\title{
Reversing the effects of early deprivation after infancy: giving children families may not be enough
}

\author{
Megan R. Gunnar* \\ Institute of Child Development, University of Minnesota, Minneapolis, MN, USA \\ *Correspondence: gunnar@umn.edu
}

\section{A commentary on}

Effects of early psychosocial deprivation on the development of memory and executive function

by Karen J. Bos, Nathan Fox, Charles H. Zeanah and Charles A. Nelson. (2009) Front. Behav. Neurosci. 3:16.

Because the human infant is born so motorically immature, for many months she must depend on caregivers to provide varied stimulation. At birth she cannot hold her head up, roll over, or even reliably bring her hands to mouth. Developing the ability to reach out and grasp objects is a skill the takes months to achieve and she will not be able to pull herself to standing, crawl or walk until the latter part of the first year. Despite this, the infant brain requires stimulation in order to develop. The importance of stimulation for the development of the visual systems has been well documented with experiments of nature (e.g., cataracts) revealing that even a month or two of degraded input during the first months of life limits the degree of expertise in processing visual input that the child will ultimately achieve (Le Grand et al., 2003). What is less well understood is how or whether early deprivation influences the development of neural systems involved in higher cognition, and additionally, if affected, whether these neural systems retain sufficient plasticity to respond to enhanced input later in development.

The importance of early stimulus deprivation in human brain development is not merely of academic interest. As Bos and colleagues note, it is of immediate relevance to the estimated 8 million children worldwide who are growing up in institutional care. Because of the popularity of adopting children from institutions in recent years, it is of practical concern to adoptive parents and to those attempting to educate these children.
Not all institutions are similarly depriving; however, because of the need to care for large numbers of infants by relatively few adults, care is typically regimented, addresses physical needs, and typically involves little response-contingent visual, tactile, auditory, linguistic, and proprioceptive stimulation.

There is a long history of studying the impact of institutional care on human development (see review, Rutter, 1981). The work of the Bucharest Early Intervention Project (BEIP) is part of a new wave of research focused on identifying the neural systems impacted by early institutional care (Behen et al., 2009; Mehta et al., 2009; Pollak et al., 2010; Tottenham et al., 2010). These other studies, however, have examined children adopted from institutions, using age at adoption as a proxy measure for duration of deprivation. What makes the BEIP work so unique and important is that it is based on random assignment of children to care as usual versus removal from the institution and placement in foster care. Thus the BEIP provides us with the only opportunity so far to isolate duration and age of exposure from characteristics of the child that might be associated with neural development and which might have affected age at adoption/ foster placement.

Bos and colleagues describe results on measures of memory and executive function assessed using the Cambridge Neuropsychological Test and Automated Battery (CANTAB) when the children were 8 years of age. This assessment largely paralleled one conducted on post-institutionalized, adopted children of comparable age in a study on which Charles Nelson also collaborated (Pollak et al., 2010). In the Pollak et al. (2010) study the postinstitutionalized group left the institution for adoption when they were 12 months or older ( $M=23.4$ months), while in the Bos et al. study, the foster care intervention group were removed from the institution and placed in foster care at 9 months or older ( $M=23.6$ months). Notably, the Pollak et al. (2010) study involved children adopted from institutions in Asia, Africa, and Latin America, as well as in Russia and Eastern Europe. The pattern of findings in the two assessments was remarkably consistent. In both studies, compared to children reared by their parents, children who spent most or all of their first year of life in institutional care showed deficits on paired associates learning, spatial working memory and measures of visual memory (Bos et al., delayed match to sample; Pollak et al., 2010, memory for faces from the NEPSY). Neither study yielded evidence of problems in spatial planning (Stockings of Cambridge). The Pollak et al. (2010) study included a group of children adopted early (by 8 months, $M=5.2$ months). Similar to results of other studies of internationally adopted children (see review, Gunnar, 2001), this early adopted group did not differ from children reared by their parents continuously from birth on any measures of memory or executive functioning.

It is a challenge to isolate effects on neurobehavioral development to postnatal conditions. Certainly it is the case that orphaned and abandoned children are likely to experience poorer prenatal conditions. It is noteworthy, however, that birth weight, which is a proxy for qualities of the prenatal environment, was only associated with one measures, and even with birth weight in the equation, Bos and colleagues still found an effect of institutional history. Unfortunately, the BEIP study did not have a group of children removed from institutional care early in infancy; this may well explain why they observed only one statistically significant effect of foster placement. Nonetheless, it was still the case that among the ever institutionalized children, placement in foster care was associated with improved scores for one measure of executive functioning. 
Taken together with other studies, Bos et al. provide evidence that early institutional deprivation impacts development of functions that depend on the medial temporal lobe and prefrontal cortex. These studies provide little support for the idea that removal from institutional care beyond a year or so of age is a sufficient intervention to ameliorate differences in functioning compared to children who never experience institutional care, although it may improve some aspects of executive function (e.g., spatial working memory). These findings have implications for the millions of infants, worldwide, who are orphaned or abandoned. Institutions are still the most common form of response to the need to care for abandoned children. Establishing and supporting orphanages is still attractive to charitable persons and organizations. Nonetheless, whatever typically happens to infants in institutions, it does not appear to reliably support development of the neural systems supporting important aspects of higher cognition.

\section{REFERENCES}

Behen, M. E., Muzik, O., Saporta, A. S., Wilson, B. J., Pai, D., Hua, J., and Chugani, H. T. (2009). Abnorma fronto-striatal connectivity in children with histories of early deprivation: a diffusion tensor imaging study. Brain Imaging Behav. 3, 292-297.

Gunnar, M. R. (2001). "Effects of early deprivation: findings from orphanage-reared infants and children," in Handbook of Developmental Cognitive Neuroscience, eds C. A. Nelson and M. Luciana (Cambridge, MA: MIT Press), 617-629.

Le Grand, R., Mondloch, C. J., Maurer, D., and Brent, H. P. (2003). Expert face processing requires visual input to the right hemisphere during infancy. Nat. Neurosci. 6, 1108-1012.

Mehta, M.A., Golembo, N. I., Nosarti, C., Colvert, E., Mota, A., Williams, S. C., Rutter, M., and Sonuga-Barke, E. J. (2009). Amygdala, hippocampal and corpus callosum size following severe early institutional deprivation: the English and Romanian Adoptees Study Pilot. J. Child Psychol. Psychiatry 50, 943-951.
Pollak, S. D., Nelson, C. A., Schlaak, M., Roeber, B., Wewerka, S., Wiik, K. L., Frenn, K.A., Loman, M. M., and Gunnar, M. R. (2010). Neurodevelopmental effects of early deprivation in post-institutionalized children. Child Dev. 81, 224-236.

Rutter, M. (1981). Maternal Deprivation Reassessed. New York: Penguin Books.

Tottenham, N.,Hare, T.A.,Quinn, B. T.,McCarry, K., Nurse, M., Gilhooly, T., Millner, A., Galvan, A. Davidson, M. C., Eigsti, I. M., Thomas, K. M., Freed P. J., Booma, E. S., Gunnar, M. R.,Altemus, M., Aronson, J., and Casey, B. J. (2010). Prolonged institutional rearing is associated with atypically larger amygdala volume and difficulties in emotion regulation. Dev. Sci. 13, 46-61.

Received: 23 July 2010; accepted: 27 August 2010; published online: 08 December 2010.

Citation: Gunnar MR (2010) Reversing the effects of early deprivation after infancy: giving children families may not be enough. Front. Neurosci. 4:170. doi: 10.3389/ fnins.2010.00170

Copyright (C) 2010 Gunnar. This is an open-access article subject to an exclusive license agreement between the authors and the Frontiers Research Foundation, which permits unrestricted use, distribution, and reproduction in any medium, provided the original authors and source are credited. 